\title{
Measurement of diamond nucleation rates from hydrocarbons at conditions comparable to the interiors of icy giant planets
}

\author{
A. K. Schuster $\odot,{ }^{1,2,{ }^{*}}$ N. J. Hartley $\odot,{ }^{1,3}$ J. Vorberger, ${ }^{1}$ T. Döppner, ${ }^{4}$ T. van Driel, ${ }^{5}$ R. W. Falcone, ${ }^{6,7}$ L. B. Fletcher, ${ }^{5}$ \\ S. Frydrych, ${ }^{4,8}$ E. Galtier $\odot,{ }^{5}$ E. J. Gamboa, ${ }^{5}$ D. O. Gericke $\odot,{ }^{9}$ S. H. Glenzer, ${ }^{5}$ E. Granados, ${ }^{5}$ M. J. MacDonald ${ }^{5,10}$ \\ A. J. MacKinnon, ${ }^{5}$ E. E. McBride, ${ }^{5,11}$ I. Nam, ${ }^{5}$ P. Neumayer, ${ }^{12}$ A. Pak, ${ }^{4}$ I. Prencipe, ${ }^{1}$ K. Voigt, ${ }^{1,2}$ \\ A. M. Saunders, ${ }^{6}$ P. Sun, ${ }^{5}$ and D. Kraus ${ }^{1,2, \dagger}$ \\ ${ }^{1}$ Institut für Strahlenphysik, Helmholtz-Zentrum Dresden-Rossendorf, 01328 Dresden, Germany \\ ${ }^{2}$ Institut für Festkörper- und Materialphysik, TU Dresden, Haeckelstraße 3, 01069 Dresden, Germany \\ ${ }^{3}$ Open and Transdisciplinary Research Institute, Osaka University, Suita, Osaka 565-0871, Japan \\ ${ }^{4}$ Lawrence Livermore National Laboratory, Livermore, California 94550, USA \\ ${ }^{5}$ SLAC National Accelerator Laboratory, Menlo Park, California 94309, USA \\ ${ }^{6}$ Department of Physics, University of California, Berkeley, California 94720, USA \\ ${ }^{7}$ Lawrence Berkeley National Laboratory, Berkeley, California 94720, USA \\ ${ }^{8}$ Institut für Kernphysik, TU Darmstadt, Schlossgartenstraße 9, 64289 Darmstadt, Germany \\ ${ }^{9}$ Centre for Fusion, Space and Astrophysics, Department of Physics, University of Warwick, Coventry CV4 7AL, United Kingdom \\ ${ }^{10}$ Applied Physics Program, University of Michigan, Ann Arbor, Michigan 48109, USA \\ ${ }^{11}$ European XFEL GmbH, Holzkoppel 4, 22869 Schenefeld, Germany \\ ${ }^{12}$ GSI Helmholtzzentrum für Schwerionenforschung GmbH, Planckstraße 1, 64291 Darmstadt, Germany
}

(Received 9 November 2018; revised manuscript received 14 November 2019; accepted 9 December 2019; published 5 February 2020)

\begin{abstract}
We present measurements of the nucleation rate into a diamond lattice in dynamically compressed polystyrene obtained in a pump-probe experiment using a high-energy laser system and in situ femtosecond x-ray diffraction. Different temperature-pressure conditions that occur in planetary interiors were probed. For a single shock reaching $70 \mathrm{GPa}$ and $3000 \mathrm{~K}$ no diamond formation was observed, while with a double shock driving polystyrene to pressures around $150 \mathrm{GPa}$ and temperatures around $5000 \mathrm{~K}$ nucleation rates between $10^{29}$ and $10^{34} \mathrm{~m}^{-3} \mathrm{~s}^{-1}$ were recorded. These nucleation rates do not agree with predictions of the state-of-the-art theoretical models for carbon-hydrogen mixtures by many orders of magnitude. Our data suggest that there is significant diamond formation to be expected inside icy giant planets like Neptune and Uranus.
\end{abstract}

DOI: 10.1103/PhysRevB.101.054301

\section{INTRODUCTION}

Icy, giant planets like Neptune and Uranus turn out to be highly abundant in our galaxy: In a recent survey it was found that there are nine times as many icy planets compared to the larger gas giants like Jupiter and Saturn [1]. Underneath their hydrogen-helium atmospheres, the interiors of these ice giants are thought to consist mainly of a dense fluid mixture of water, methane, and ammonia [2]. This material mix likely undergoes chemical reactions and structural transitions due to the high-pressure, high-temperature conditions deep inside the planets' interiors [3-5]. A prominent example of such a reaction is the possible carbon-hydrogen dissociation and the

\footnotetext{
*a.schuster@hzdr.de

†d.kraus@hzdr.de
}

Published by the American Physical Society under the terms of the Creative Commons Attribution 4.0 International license. Further distribution of this work must maintain attribution to the author(s) and the published article's title, journal citation, and DOI. subsequent phase separation that results in the formation of diamonds [6-8]. The diamonds are denser than the surrounding fluid and therefore precipitate towards the planetary centers [3]. The precipitation releases enough gravitational energy to act as an energy source that significantly contributes to the energy balance of the planet [5]. The phase-separation reaction may also result in the formation of localized metallic hydrogen [9], which, apart from possible superionic phases of water and ammonia [10], contributes to a conducting region which may play a key role in the generation of the unusual magnetic fields observed for both Neptune and Uranus [11,12].

However, the latest theoretical calculations based on molecular dynamics (MD) simulations and classical nucleation theory have predicted that nucleation rates into a diamond lattice in the planetary environment may be extremely low, resulting in no diamond formation within Neptune and Uranus over the lifetime of the universe [13,14]. Thanks to new experimental capabilities, chemical activity can be observed in situ at conditions comparable to the deep interiors of the ice giants and significant diamond formation from polystyrene, $\left(\mathrm{C}_{8} \mathrm{H}_{8}\right)_{n}$, at $\sim 150 \mathrm{GPa}$ and $\sim 5000 \mathrm{~K}$ was ob- 


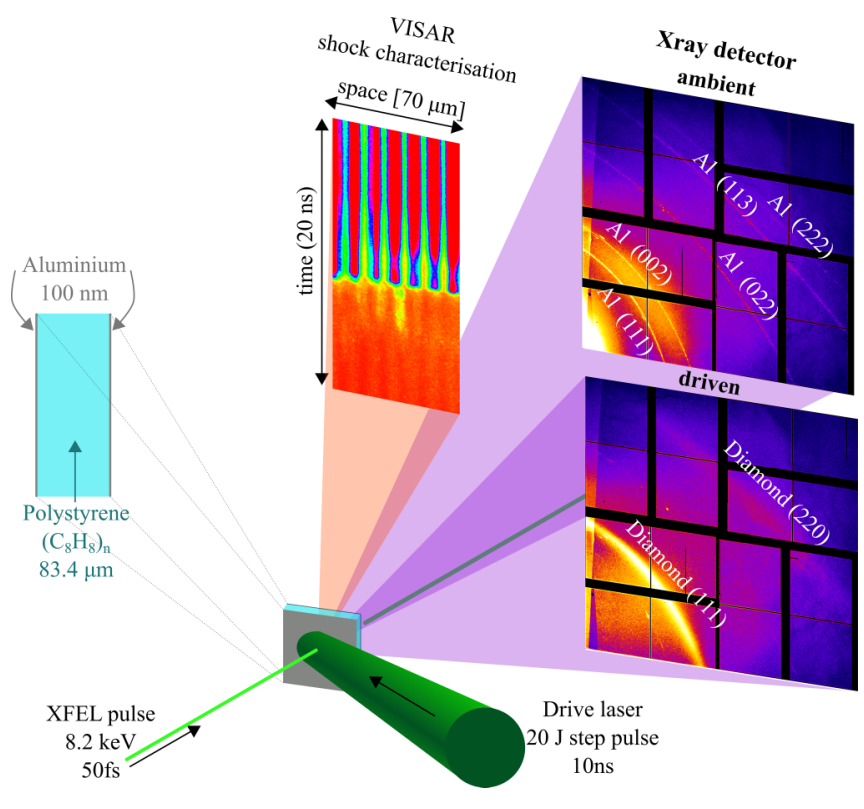

FIG. 1. Schematic setup of the experiment at the MEC end station at LCLS. Raw data at ambient and driven conditions at roughly the time of shock coalescence at the rear side of the sample are presented. The corresponding line-outs are displayed in Fig. 2.

served [6]. In this article, we infer the amount of carbon atoms transferred to diamond structures in these experiments. We can then estimate nucleation rates into a diamond lattice that are more than 100 orders of magnitude greater than suggested by the theoretical model mentioned above. Moreover, we find a highly different trend for varying temperature and pressure conditions. Therefore, new models for diamond nucleation in planetary interiors need to be developed.

\section{EXPERIMENT}

We performed the experiments at the Matter in Extreme Conditions (MEC) end station of the Linac Coherent Light Source (LCLS) of the SLAC National Accelerator Laboratory $[15,16]$. A schematic of the experimental setup is shown in Fig. 1. The hydrocarbon samples were made out of 83.4- $\mu \mathrm{m}$-thick polystyrene foils and were coated with 100 $\mathrm{nm}$ aluminum on both sides; the front layer prevented the interaction with laser prepulses and the rear coating was needed for the velocity interferometer system for any reflector (VISAR) diagnostic to determine shock parameters [17]. A high-energy laser system generated two subsequent shock waves in the polystyrene that were designed to coalesce on the rear side of the sample in order to generate the temperature and pressure conditions found in the interiors of icy giants. Structural changes could be observed by in situ X-ray diffraction (XRD) with the LCLS pulses of $8.2 \mathrm{keV}$ photon energy and $50 \mathrm{fs}$ pulse duration. Three different drive pulse shapes were realized reaching $150 \pm 15 \mathrm{GPa}$ and $5000 \pm 500 \mathrm{~K}$ (intermediate drive), $159 \pm 15 \mathrm{GPa}$ and $6100 \pm 500 \mathrm{~K}$ (high drive), and $139 \pm 15 \mathrm{GPa}$ and $4200 \pm 500 \mathrm{~K}$ (low drive), respectively. A more detailed description of the experimental method, including detailed hydrodynamic simulations and a

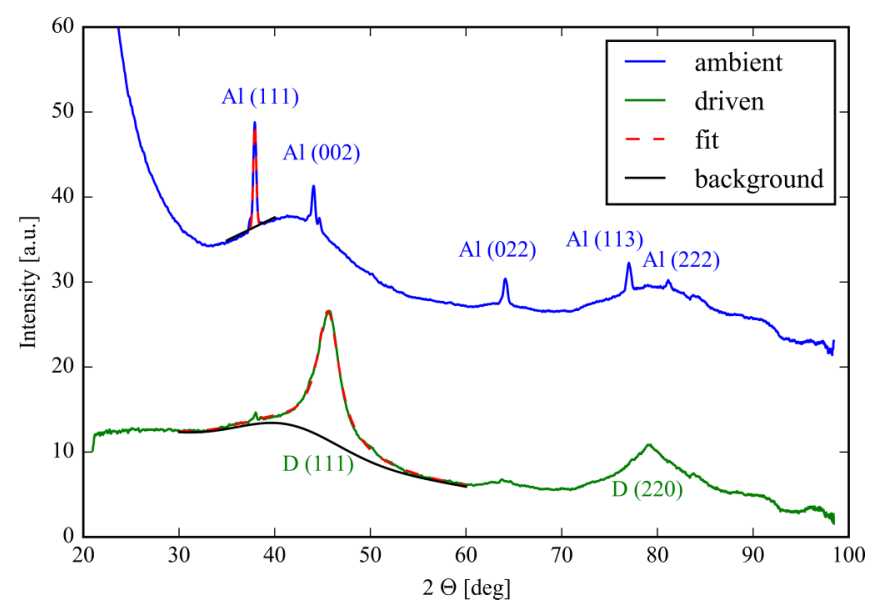

FIG. 2. Line-outs of the diffraction data in Fig. 1 with corresponding fit functions for the (111) peaks of aluminum and diamond, respectively. The driven case corresponds to the intermediate drive conditions: $150 \pm 15 \mathrm{GPa}$ and $5000 \pm 500 \mathrm{~K}$ (see first row in Table I).

discussion of the pressure and temperature conditions reached in the experiment, can be found in Refs. [6,7].

Raw data from X-ray diffraction showing images from polystyrene at ambient conditions and from a driven sample at the time of approximate shock coalescence at the sample rear side are shown in Fig. 1. Via azimuthal integration the corresponding line-outs are obtained (see Fig. 2), taking into account the horizontal polarization of the x-ray laser and the detector geometry. For the samples at ambient conditions, the diffraction signatures of both the amorphous polystyrene (the blue line-out subtracting the sharp Al Bragg peaks) as well as the thin aluminum coatings (Bragg peaks in the blue line-out) can be observed. In the driven case, the formation of compressed diamond crystallites is clearly visible, as demonstrated by the appearance of the corresponding (111) powder diffraction ring above a broader diffraction signature of a remaining warm dense $\mathrm{CH}$ liquid [7]. Complying with the assumption that diamond crystallites are formed in quasi-steady-state conditions after the second shock wave has passed, we do not find evidence for a preferred orientation of the crystallites.

\section{RESULTS}

In order to infer the number of carbon atoms that form diamond crystal lattices inside the sample volume, we compare the observed intensity of the diamond (111) diffraction ring $I_{D, \text { expt }}^{(111)}$ to the diffraction from the two aluminum layers of well-known thickness. We use the aluminum (111) reflection intensity, $I_{\mathrm{Al} \text {,expt }}^{(111)}$, for normalization, as it is strong in signal and thus promotes uncertainty reduction. Additionally, the proximity to the diamond (111) reflection reduces the influence of geometric effects and possible detector sensitivity variations with changing scattering angle. The Al (111) intensity is obtained by fitting a Gaussian function and subtracting a locally linear background; for the diamond (111) intensity a Lorentzian function over a combination of linear and Gaussian background was modeled. Examples of corresponding fits can 
TABLE I. The fraction of carbon atoms having formed diamonds, $f_{D}$, the in situ measured high-pressure density $\rho_{D}$ of the nanodiamonds, as well as a value range for the nucleation rate into a diamond lattice from $R_{D, \text { low }}^{100 \mathrm{~m}}$ to $R_{D \text {, up }}^{4 n \mathrm{~m}}$ assuming an average crystallite size of 100 and $4 \mathrm{~nm}$, respectively.

\begin{tabular}{lcccc}
\hline \hline$f_{D}$ & $\rho_{D}$ & $R_{D, \mathrm{low}}^{100 \mathrm{~nm}}$ \\
$(\%)$ & $\left(\mathrm{g} / \mathrm{cm}^{3}\right)$ & $\left(\times 10^{30} \mathrm{~m}^{-3} \mathrm{~s}^{-1}\right)$ & $\begin{array}{c}R_{D, \mathrm{up}}^{4 \mathrm{~nm}} \\
\left(\times 10^{34} \mathrm{~m}^{-3} \mathrm{~s}^{-1}\right)\end{array}$ & Drive \\
\hline $60 \pm 11$ & $4.13 \pm 0.07$ & 1.8 & 2.8 & Intermediate \\
$38 \pm 7$ & $4.15 \pm 0.05$ & 1.0 & 1.5 & Intermediate \\
$45 \pm 5$ & $3.97 \pm 0.08$ & 1.4 & 2.2 & Low \\
$51 \pm 10$ & $4.08 \pm 0.05$ & 1.4 & 2.2 & Low \\
$35 \pm 5$ & $4.23 \pm 0.05$ & 0.9 & 1.4 & High \\
\hline \hline
\end{tabular}

be seen in Fig. 2. With these data, the total number of carbon atoms in a diamond lattice can be estimated by

$$
N_{C, D}=2 \frac{I_{D, \text { expt }}^{(111)}}{I_{\mathrm{Al} \text {,expt }}^{(111)}} \frac{I_{A l, u c}^{(111)}}{I_{D, u c}^{(111)}} N_{A l},
$$

where $I_{C, u c}^{(111)}$ and $I_{A l, u c}^{(111)}$ denote the theoretical intensities for the respective unit cells, which in the case of diamond contains two times more atoms than aluminum and $N_{A l}$ is the known number of aluminum atoms inside the sample volume. The theoretical intensities were calculated according to

$$
I_{u c}^{h k l}=\left|F_{h k l}\right|^{2} P_{L} M,
$$

where $F_{h k l}, P_{L}$, and $M$ are the structure factor, the Lorentzpolarization factor, and the multiplicity, respectively [18]. Additionally, damping of the Bragg peak intensities is taken into account. Thermal lattice vibrations contribute most dominantly by reducing the intensity of the crystal plane reflections by a factor of $e^{-2 W}$, where $2 W$ is known as the Debye-Waller factor $[19,20]$. At a diffraction angle of $k=3.2 \AA^{-1}, 2 W$ has been calculated to be on the order of 0.13 by density functional theory (DFT)-MD simulations of diamond at $150 \mathrm{GPa}$ and $5000 \mathrm{~K}$ (see Appendix A and Refs. [20-29]). For the aluminum (111) peak no such correction is necessary since the XRD pattern was recorded at ambient conditions.

Comparing the number of carbon atoms that form diamond crystal lattices with the overall number of carbon atoms inside the sample volume, $N_{C, 0}$, provides the fraction of carbon atoms $f_{D}=N_{C, D} / N_{C, 0}$ that have formed diamond crystals. In our experiments, we find $f_{D}$ to reach values of up to $\sim 60 \%$ (see Table I). The provided uncertainties of $f_{D}$ are dominated by the flexibility in setting the background while fitting the diamond (111) diffraction peak. Performing this procedure with different Bragg reflections, e.g., $\mathrm{Al}$ (002) and diamond (111), shows very similar amounts but exhibits significantly larger uncertainties due to the lower signal intensity. The in situ diamond density uncertainty $\Delta \rho_{D}$ is based on error propagation with respect to $2 \Theta$ where $\Delta(2 \Theta)$ corresponds to the standard deviation of the center of the fitted peak.

To estimate the number of diamonds formed in the sample volume requires knowledge of the average size of the crystallites. The latter quantity can be estimated by the width of the diffraction peak using the Scherrer equation [30]

$$
L_{D}=\frac{K \lambda}{\Delta(2 \theta) \cos \theta},
$$

where $L_{D}$ is the crystal size, $K$ is a shape factor which is 0.89 for spheres [30], $\Delta(2 \theta)$ is the peak width (FWHM), and $\theta$ is the Bragg angle. In this case, the Scherrer formula can only provide a lower estimate for the crystallite size since both crystal defects and density gradients inside the sample also result in peak broadening. In our experiments, we obtain an average lower limit of the diamond crystallite size of $4 \mathrm{~nm}$. In general, crystallite sizes smaller than $100 \mathrm{~nm}$ are determined by the line profile analysis by Scherrer, while crystallites between 0.1 and $100 \mu \mathrm{m}$ are measured by methods designed to analyze spotty two-dimensional (2D) diffraction patterns [31]. Thus, a conservative upper limit of $100 \mathrm{~nm}$ can be estimated given that no distinct spots can be observed in the Debye rings. Likely, the upper limit is significantly smaller, but, in this respect, the Scherrer analysis cannot provide fully conclusive results. From those values, the number density of nanodiamonds inside the sample volume is calculated using

$$
n_{D}=n_{C} f_{D} \frac{6 m_{C}}{\pi L_{D}^{3} \rho_{D}},
$$

where $n_{C}=\rho_{C H} /\left(m_{C}+m_{H}\right)$ is the total number density of the carbon atoms inside a compressed polystyrene sample with density $\rho_{C H}$ and atomic masses $m_{C}$ for carbon and $m_{H}$ for hydrogen. The density of the diamonds, $\rho_{D}$, at the highpressure conditions in the experiment (see Table I) can be inferred in situ by the angular position of the (111) Bragg reflection. Depending on the time delay between the pump and probe and the energy of the shot, densities between 3.97 and $4.23 \mathrm{~g} / \mathrm{cm}^{3}$ were reached. With these data, we find diamond number densities between $1 \times 10^{25}$ and $6 \times 10^{20} \mathrm{~m}^{-3}$ for assumed crystallite sizes of 4 and $100 \mathrm{~nm}$, respectively.

In order to obtain a nucleation rate into a diamond lattice, $R_{D}$, from this result, we need to consider the time scale of the experiment. The first compression wave (e.g., $\sim 60 \mathrm{GPa}$, $\sim 4000 \mathrm{~K}$, for the intermediate drive) takes $\sim 7.5 \mathrm{~ns}$ to reach the sample rear side. The second compression wave that creates the high-pressure, high-temperature conditions required for diamond formation is launched $6 \mathrm{~ns}$ after the initial shock and traverses the precompressed sample volume within $\sim 1 \mathrm{~ns}$ $[6,7]$. Therefore, there were on average $t_{D}=500 \mathrm{ps}$ to form diamonds at the moment when the two compression waves overlap at the sample rear side. For the nucleation rate into a diamond lattice given as

$$
R_{D}=\frac{n_{D}}{t_{D}}
$$




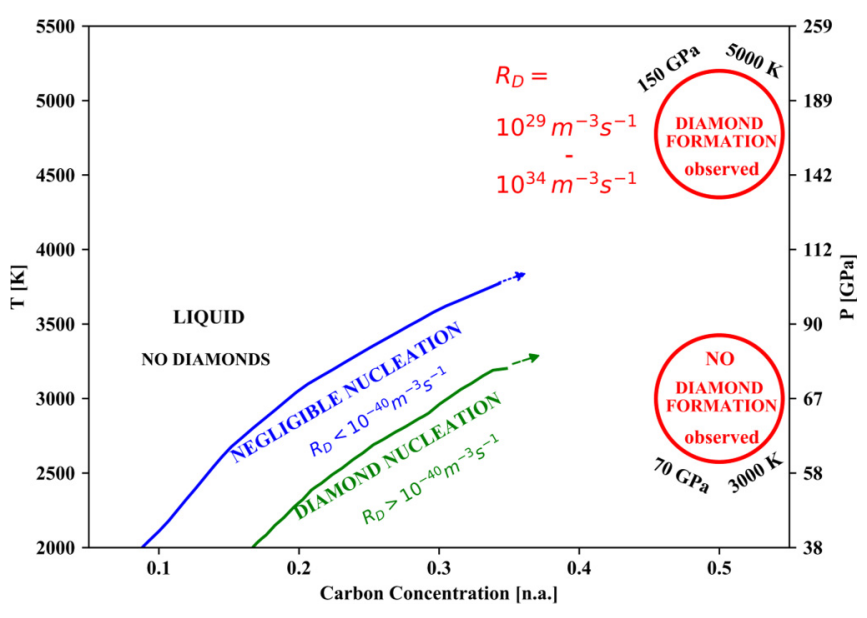

FIG. 3. The diamond nucleation boundary as a function of carbon concentration based on the work of Refs. $[13,14]$ is shown along with the results of our study. The temperature and the pressure axes are related along the Uranus isentrope [32]. In the liquid, $R_{D}$ is zero; in the negligible region, it is $R_{D}<10^{-40} \mathrm{~m}^{-3} \mathrm{~s}^{-1}$; and in the diamond nucleation region, $R_{D}>10^{-40} \mathrm{~m}^{-3} \mathrm{~s}^{-1}$. The blue and green arrows indicate the trend for those regions with higher dilution values following the scaling given in Ref. [13]. Our data points are marked with red circles.

we obtain nucleation rates between $10^{29}$ and $10^{34} \mathrm{~m}^{-3} \mathrm{~s}^{-1}$. The data for five data sets are shown in Table I. The listed values are expected to be sensitive to multiple parameters such as laser energy, time profile of the pump laser, and time delay between the pump and the probe laser. The amount of ablated material is negligibly small and was therefore not considered. Moreover, X-ray intensity fluctuations between shots were taken into account for the $\mathrm{Al}$ (111) peak at ambient conditions and for the diamond (111) peak at driven conditions.

\section{DISCUSSION}

With a single shock reaching conditions around $70 \mathrm{GPa}$ and $3000 \mathrm{~K}$ no diamond formation was observed in our experiment, whereas with a double shock reaching $150 \mathrm{GPa}$ and $5000 \mathrm{~K}$ a strong signal was recorded. A comparison of our results with the prediction by Ghiringhelli et al. $[13,14]$ is depicted in Fig. 3. In general, we find no agreement, which is a clear indication that a new theory for diamond nucleation needs to be developed. In particular, we observe rapid diamond formation at conditions where the prediction suggests nucleation rates below a numerical noise limit of $10^{-300} \mathrm{~m}^{-3} \mathrm{~s}^{-1}[13,14]$. This rate is several hundred orders of magnitude lower than observed in the experiment. Moreover, we find no diamond formation at conditions where the predictions suggest rapid diamond formation on the nanosecond time scale of our experiment. For the model system of polystyrene explored under experimental conditions similar to that expected to be found within the interiors of icy giant planets, the model approach of using nucleation rates into a diamond lattice determined by MD simulations for supercooled pure carbon in combination with classical nucleation theory to construct the mixture and concentration effects does not correctly derive the nucleation rates observed. This may be a result of elemental separation that takes place between $\mathrm{C}$ and $\mathrm{H}$ under our experimental conditions, or a fundamental flaw in the approach of using classical nucleation theory to describe this system.

Indeed, the approach of Ghiringhelli et al. does not account for local fluctuations of the carbon concentration or for chemical properties of hydrogen, oxygen, and nitrogen as other constituents of the material mix. In fact, the insulator-metal transition of hydrogen [33] may result in the immiscibility of carbon in metallic hydrogen. Moreover, first-principles MD studies suggest that the presence of both oxygen and nitrogen more supports than prevents the formation of isolated carbon clusters at conditions comparable to planetary interiors [5]. To emphasize, our data account only for the presence of hydrogen, but ongoing work is investigating materials better fitting the planetary mix with more constituents. In particular, we are conducting additional experiments to probe the role that nitrogen and oxygen may play in the chemical system. Furthermore, it cannot be excluded that a stepwise sequence of partial decomposition of polystyrene into hydrocarbons and diamond precedes a complete phase separation into the elemental constituents [34].

\section{CONCLUSIONS}

In conclusion, the diamond formation rates observed in our experiments investigating the simplified $\mathrm{CH}$ system using polystyrene as initial material suggest that there is potentially significant carbon precipitation inside icy giant planets like Neptune and Uranus. Under the very simplistic assumption that the pressure and temperature conditions during precipitation allow the diamond growth rate to stay constant over time, the formation of diamonds of $4 \mathrm{~nm}$ in diameter within 500 ps can be linearly extrapolated to diamonds of $70 \mathrm{~cm}$ in diameter within 100 million years. As this does not include accumulation of carbon particles, this may be seen as a lower estimate. This would result in a thick carbon layer formed by diamond precipitation around the rocky cores of Uranus, Neptune, and other comparable planets. Conclusive experiments that investigate samples closer to the predicted HCNO stoichiometry in the icy giants are under way.

\section{ACKNOWLEDGMENTS}

This work was performed at the Matter in Extreme Conditions (MEC) instrument of LCLS, supported by the U.S. Department of Energy Office of Science, Fusion Energy Science, under Contract No. SF00515. A.K.S., N.J.H., K.V., and D.K. are supported by the Helmholtz Association under VH-NG-1141. R.W.F., M.J.M., and A.M.S. were supported by DOE, Office of Science, Office of Fusion Energy Sciences under Contract No. DE-SC0018298, and through the University of California, Center for High Energy Density Science. A.M.S. was supported by the Department of Energy National Nuclear Security Administration Stewardship Science Graduate Fellowship program, which is provided under Grant No. DE-NA002135. SLAC HED is supported by DOE Office of Science, Fusion Energy Science under FWP 100182. S.F. was supported by German Bundesministerium für Bildung und Forschung Project No. 05P15RDFA1. The work of A.P., S.F., 

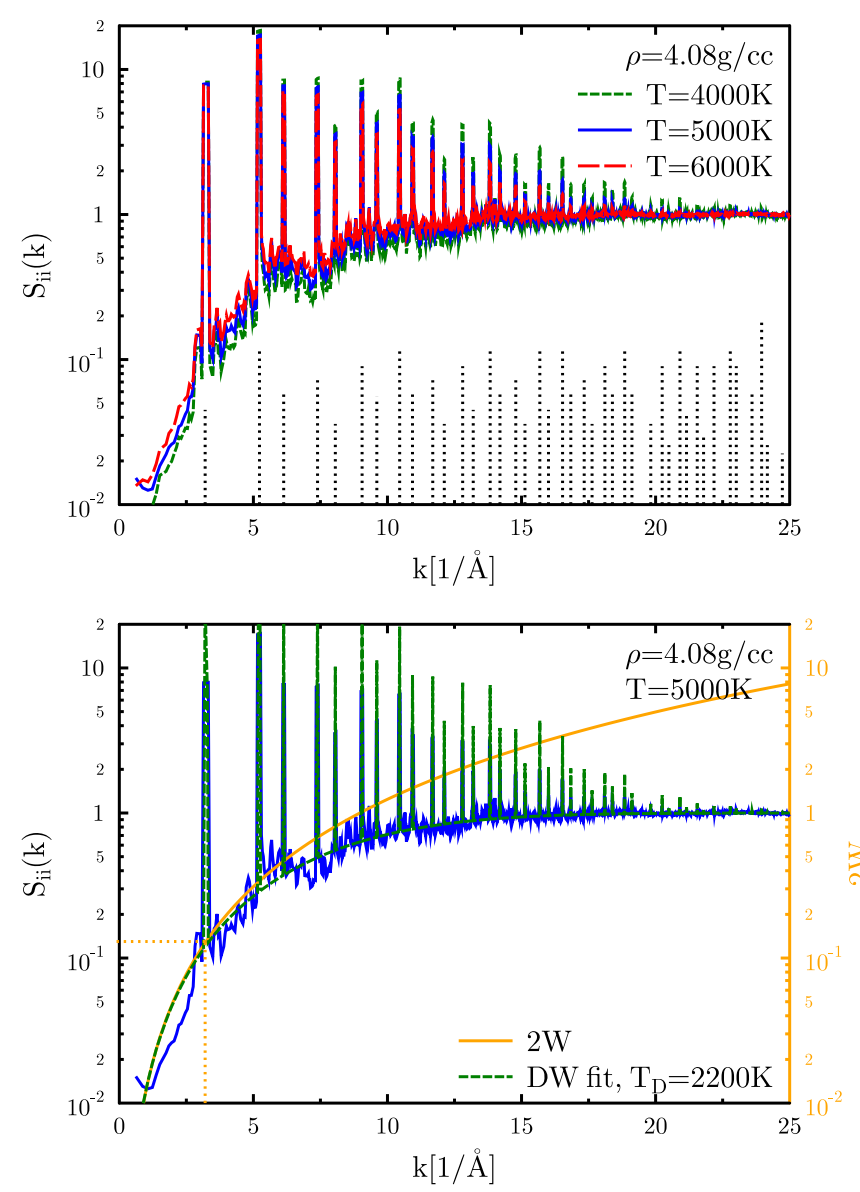

FIG. 4. The static ion structure factor for diamond from DFTMD at a density of $\rho=4.08 \mathrm{~g} / \mathrm{cm}^{3}$ and several different temperatures. The temperature dependence is given in the top panel. In the bottom panel, the fit of the DFT-MD structure factor with the Debye-Waller model is given. The used wave number of $k=3.2 / \AA$ is indicated.

and T.D. was performed under the auspices of the US Department of Energy by Lawrence Livermore National Laboratory under Contract No. DE-AC52-07NA27344.

\section{APPENDIX A: DFT SIMULATIONS}

All DFT-MD simulations were performed using the VASP package, version 5.2 [21-24]. The electronic density in the simulation box with periodic boundary conditions was represented by a plane-wave expansion with a cutoff energy of $E_{\text {cut }}=1000 \mathrm{eV}$. We used the Mermin formulation of DFT to optimize the Helmholtz free energy at a given temperature [25]. The electron-ion interaction was modeled using the projector-augmented wave (PAW) approach, specifically the hard PAW pseudopotentials for carbon (four valence electrons, C_h Feb 2004) as provided with VASP [26,27]. The exchange-correlation potential was taken in the generalized gradient approximation in Perdew-Burke-Ernzerhof parametrization (GGA-PBE) [28]. We generally sampled the Brillouin zone of the supercell at the $\Gamma$ point only. The electronic bands were populated using a Fermi distribution at the chosen temperature. The supercell contained 216 atoms

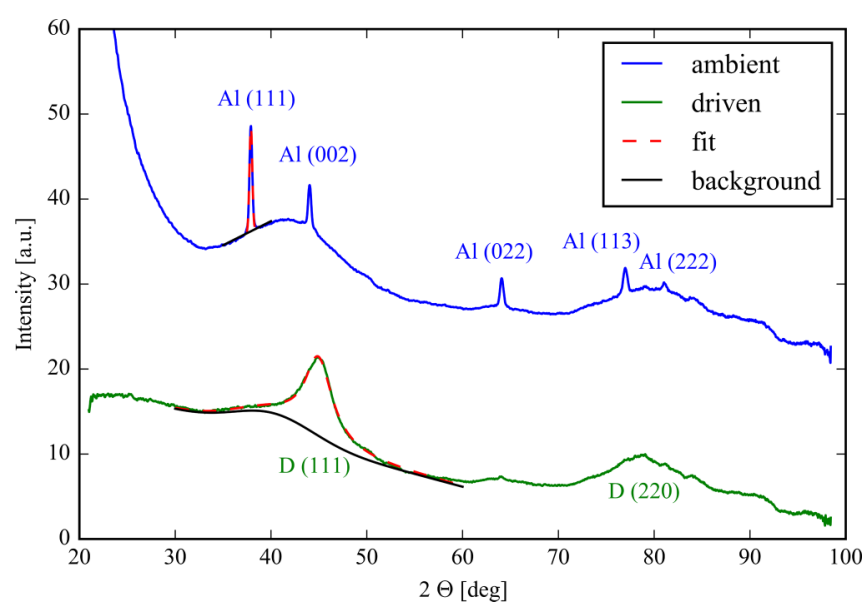

FIG. 5. Line-out intermediate drive conditions with $f_{D}=$ $(38 \pm 7) \%$.

of carbon in a diamond lattice, and their movements were calculated using the Hellman-Feynman forces derived from the electron densities of DFT under the Born-Oppenheimer approximation. The time step was $t=0.2$ fs and the DFT-MD run covered a time span of $2-4$ ps. The ion temperature was controlled by a Nosè-Hoover thermostat [29]. From the recorded coordinates, the ion structure, and therefore the intensity of the Bragg peaks and the diffuse scattering background, can be obtained.

According to Eq. (19) of Gregori et al. [20], the structure in a solid can be expressed as

$$
S_{i i}(k)=S^{\text {plasma }}(k)\left[\left(1-e^{-2 W}\right)+e^{-2 W} b(k)\right],
$$

where $S^{\text {plasma }}$ is a factor describing plasma structure contributions. $2 W$ is the Debye-Waller factor that produces a diffuse background signal $1-\exp (-2 W)$ and damps the Bragg peaks $b(k)$. We can use the structure from DFT-MD simulations to extract the Debye-Waller factor which is parametrized using the effective Debye temperature $T_{D}$. Under the condition $S^{\text {plasma }}(k)=1$ it follows that

$$
2 W=-\ln \frac{1-S_{i i}^{\mathrm{DFT}}(k)}{1-b(k)}
$$

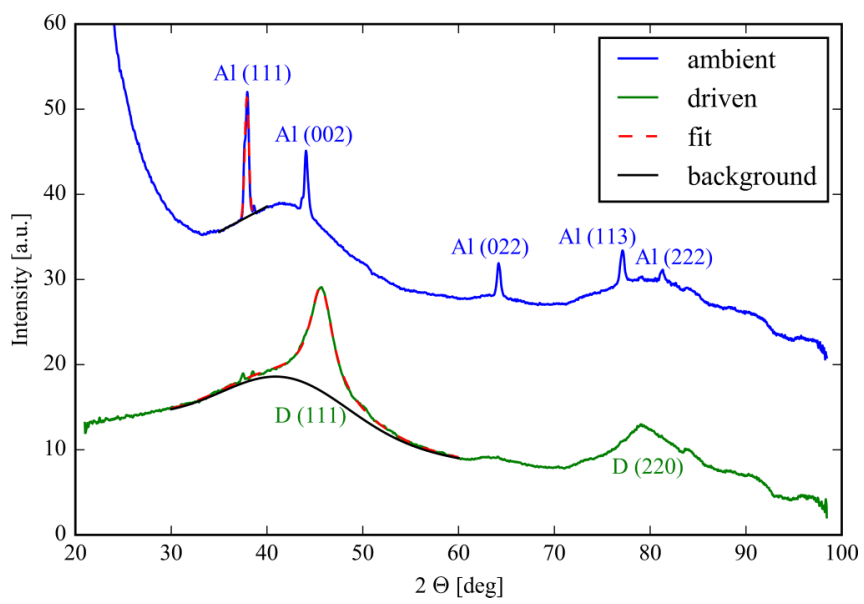

FIG. 6. Line-out low drive conditions with $f_{D}=(45 \pm 5) \%$. 


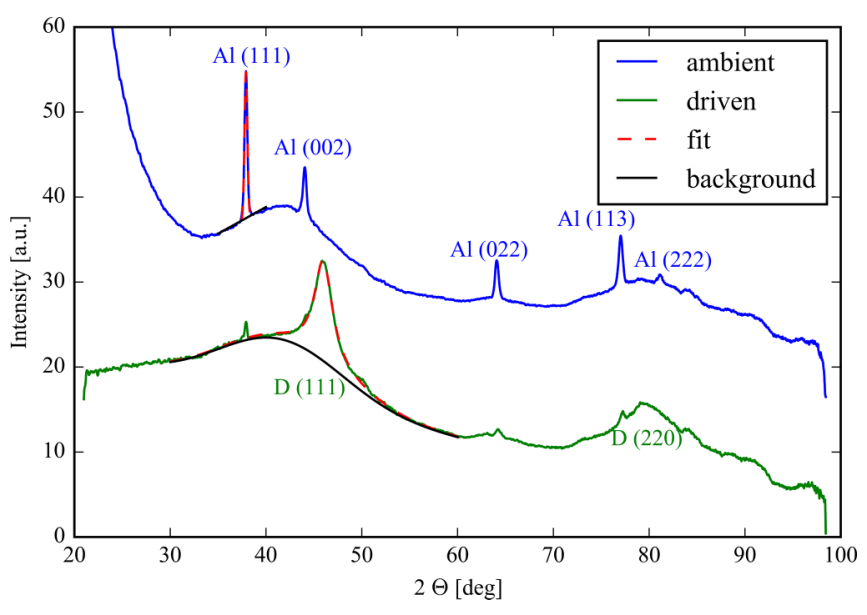

FIG. 7. Line-out low drive conditions with $f_{D}=(51 \pm 10) \%$.

Using Debye theory of the phonons, one derives

$$
2 W=\frac{\hbar^{2} k^{2}}{2 M} \frac{3 N}{\omega_{D}^{3}} \int_{0}^{\omega_{D}} d \omega \omega\left[\frac{2}{e^{\beta \hbar \omega}-1}+1\right]
$$

and

$$
2 W=\frac{\hbar^{2} k^{2}}{2 M} \frac{3 N}{\omega_{D}}\left[\frac{2 k_{B} T}{\omega_{D}} D_{1}\left(\frac{\omega_{D}}{k_{B} T}\right)+\frac{1}{2}\right]
$$

with $\omega_{D}=k_{B} T_{D}$ and $D_{1}(x)=1 / x \int_{0}^{x} d t t /(\exp (t)-1)$. In special limits for the temperature with respect to the Debye

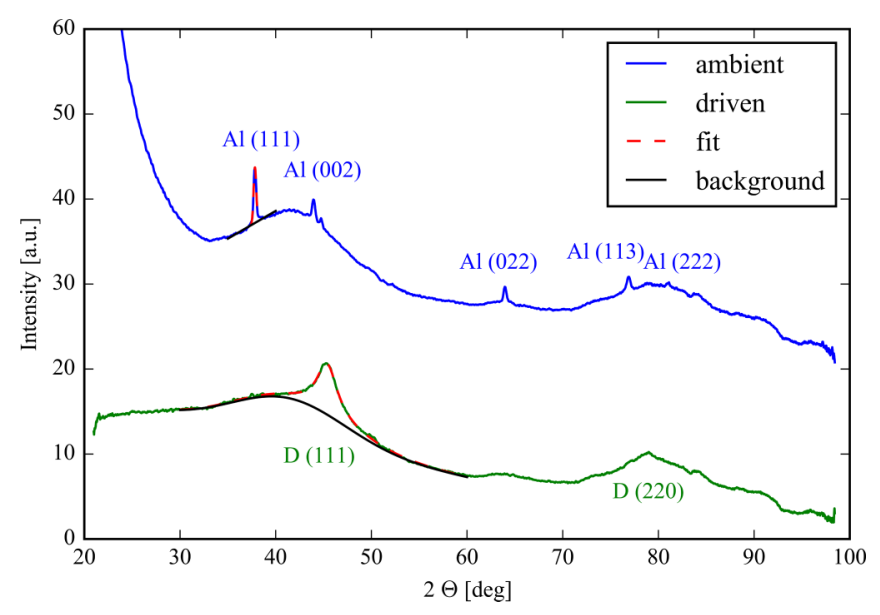

FIG. 8. Line-out high drive conditions with $f_{D}=(35 \pm 5) \%$.

temperature, one obtains

$$
\begin{gathered}
2 W=\frac{6 \hbar^{2} k^{2} \hbar^{2}}{2 M k_{B} T_{D}}\left(\frac{T}{T_{D}}\right) \quad \forall T_{D} \ll T, \\
2 W=\frac{\pi^{2} \hbar^{2} k^{2}}{2 M k_{B} T_{D}}\left(\frac{T}{T_{D}}\right)^{2}+\frac{3 \hbar^{2} k^{2}}{4 M k_{B} T_{D}} \forall T_{D} \gg T .
\end{gathered}
$$

In Fig. 4, the extraction of the Debye-Waller factor, respectively the Debye temperature, from the DFT-MD static structure factor for the experimental conditions is shown.

\section{APPENDIX B: LINE-OUTS FOR ALL PRESENTED DATA SETS}

The line-outs corresponding to the data sets presented in rows 2-5 in Table I are displayed in Figs. 5-8, respectively.
[1] W. J. Borucki, Rep. Prog. Phys. 79, 036901 (2016).

[2] T. Guillot and D. Gautier, in Treatise on Geophysics, edited by G. Schubert (Elsevier, Amsterdam, 2015), Chap. 10, pp. 529-557.

[3] M. Ross, Nature (London) 292, 435 (1981).

[4] S. S. Lobanov, P.-N. Chen, X.-J. Chen, C.-S. Zha, K. D. Litasov, H.-K. Mao, and A. F. Goncharov, Nat. Commun. 4, 2446 (2013).

[5] R. Chau, S. Hamel, and W. J. Nellis, Nat. Commun. 2, 203 (2011).

[6] D. Kraus, J. Vorberger, A. Pak, N. Hartley, L. Fletcher, S. Frydrych, E. Galtier, E. Gamboa, D. Gericke, S. Glenzer et al., Nat. Astron. 1, 606 (2017).

[7] D. Kraus, N. Hartley, S. Frydrych, A. Schuster, K. Rohatsch, M. Rödel, T. Cowan, S. Brown, E. Cunningham, T. van Driel et al., Phys. Plasmas 25, 056313 (2018).

[8] L. R. Benedetti, J. H. Nguyen, W. A. Caldwell, H. Liu, M. Kruger, and R. Jeanloz, Science 286, 100 (1999).

[9] J. M. McMahon, M. A. Morales, C. Pierleoni, and D. M. Ceperley, Rev. Mod. Phys. 84, 1607 (2012).

[10] M. Bethkenhagen, D. Cebulla, R. Redmer, and S. Hamel, J. Phys. Chem. A 119, 10582 (2015).
[11] W. Nellis, in Shock Compression of Condensed Matter - 2015: Proceedings of the Conference of the American Physical Society Topical Group on Shock Compression of Condensed Matter, AIP Conf. Proc. No. 1793 (AIP, New York, 2017), p. 090002.

[12] R. Redmer, T. R. Mattsson, N. Nettelmann, and M. French, Icarus 211, 798 (2011).

[13] L. M. Ghiringhelli, C. Valeriani, E. J. Meijer, and D. Frenkel, Phys. Rev. Lett. 99, 055702 (2007).

[14] L. M. Ghiringhelli, C. Valeriani, J. Los, E. J. Meijer, A. Fasolino, and D. Frenkel, Mol. Phys. 106, 2011 (2008).

[15] B. Nagler, B. Arnold, G. Bouchard, R. F. Boyce, R. M. Boyce, A. Callen, M. Campell, R. Curiel, E. Galtier, J. Garofoli et al., J. Synchrotron Radiat. 22, 520 (2015).

[16] S. Glenzer, L. Fletcher, E. Galtier, B. Nagler, R. Alonso-Mori, B. Barbrel, S. Brown, D. Chapman, Z. Chen, C. Curry et al., J. Phys. B: At. Mol. Opt. Phys. 49, 092001 (2016).

[17] L. Barker and R. Hollenbach, J. Appl. Phys. 43, 4669 (1972).

[18] CrystalDiffract User's Guide, distributed with "CrystalDiffract 6", a computer program for powder diffraction simulation and analysis (CrystalMaker Software Ltd, Oxford, England), http: //crystalmaker.com. 
[19] V. Sears and S. Shelley, Acta Crystallogr. Sect. A 47, 441 (1991).

[20] G. Gregori, S. H. Glenzer, and O. L. Landen, Phys. Rev. E 74, 026402 (2006).

[21] G. Kresse and J. Hafner, Phys. Rev. B 47, 558 (1993).

[22] G. Kresse and J. Hafner, Phys. Rev. B 49, 14251 (1994).

[23] G. Kresse and J. Furthmüller, Comput. Mater. Sci. 6, 15 (1996).

[24] G. Kresse and J. Furthmüller, Phys. Rev. B 54, 11169 (1996).

[25] N. D. Mermin, Phys. Rev. 137, A1441 (1965).

[26] P. E. Blöchl, Phys. Rev. B 50, 17953 (1994).

[27] G. Kresse and D. Joubert, Phys. Rev. B 59, 1758 (1999).
[28] J. P. Perdew, Phys. Rev. Lett. 78, 1396 (1997).

[29] S. Nosé, Prog. Theor. Phys. Suppl. 103, 1 (1991).

[30] A. Monshi, M. R. Foroughi, and M. R. Monshi, World J. Nano Sci. Eng. 2, 154 (2012).

[31] B. B. He, Two-Dimensional X-Ray Diffraction (Wiley, New York, 2018).

[32] S. Scandolo, G. Chiarotti, and E. Tosatti, Phys. World 13, 31 (2000).

[33] P. M. Celliers, M. Millot, S. Brygoo, R. S. McWilliams, D. E. Fratanduono, J. R. Rygg, A. F. Goncharov, P. Loubeyre, J. H. Eggert, J. L. Peterson et al., Science 361, 677 (2018).

[34] L. J. Conway and A. Hermann, Geosciences 9, 227 (2019). 